\title{
$b$ decays: A factory for hidden charm multiquark states
}

\author{
F. Buccella \\ Istituto Nazionale di Fisica Nucleare, via Cinthia 80126 Sezione di Napoli, Napoli, Italy
}

(Received 21 June 2018; published 13 December 2018)

\begin{abstract}
We assume that the two hidden charm pentaquark states discovered at $\mathrm{LHCb}$ are built from three light quarks and a $c \bar{c}$ pair. Further assumed is that the three light quarks and the $c \bar{c}$ pair are both in colour octet states. Thus, for the final $J^{P}=\frac{5}{2}^{+}$state, the three light quarks and the $c \bar{c}$ pair are in a relative P-state, whereas the five constituents are in a relative S-state forming the four $J^{P}=\frac{3}{2}^{-}$states, the four eigenstates of the chromomagnetic Hamiltonian with masses 4360, 4409, 4491 and $4560 \mathrm{MeV}$. The "open channel" $[p+J / \psi]$ has large components along the two lower mass states and they thus appear as a resonance with a mass $4380 \mathrm{MeV}$, whereas $\left[\Lambda_{c} \bar{D}^{*}\right]$ and $\left[\Sigma_{c} \bar{D}^{*}\right]$ are "open channels" respectively for the two lowest and the two highest mass resonances, respectively. The small width of the $5 / 2^{+}$state is due to the fact that its decay requires one-gluon exchange. The decays of particles with beauty furnish the best experimental technique for discovering multiquark states with hidden charm. Also, the mechanism of production proposed here explains why only a few states with nonminimal constituents have been discovered so far.
\end{abstract}

DOI: $10.1103 /$ PhysRevD.98.114011

\section{INTRODUCTION}

The discovery of two pentaquarks with hidden charm in the decay [1]:

$$
\Lambda_{b} \rightarrow p+J / \psi+K^{-}
$$

confirms the propensity of particles with beauty to give rise to multiquark states with hidden charm, as previously seen [2] for the $\left(3872,1^{+}\right)$resonance decaying into $J / \psi+\rho^{0}$ (or $\omega$ ) along with a kaon in the decay of $B_{q}$ 's. The study of states with nonminimal number of constituents began about fifty years ago:

(i) for $2 q 2 \bar{q}$ states, see [3-5];

(ii) for $6 q$ states, see $[6,7]$;

(iii) and, last but not least, for $4 q \bar{q}$ states, see [8]. Their existence has been confirmed through the analysis that confirmed the existence of the $\left[\Theta^{+}, Y=2\right.$ baryon resonance] [9] and by the large cross section at high momentum transfer for the production of $\left(3872,1^{+}\right)$ resonance [10], which shows that it is a compact object [11] and not a molecule, that should behave as the deuteron; and, as such, should be very rarely produced at high momenta [12]. This seems to confirm the $\left[\begin{array}{ll}2 q & 2 \bar{q}\end{array}\right]$

Published by the American Physical Society under the terms of the Creative Commons Attribution 4.0 International license. Further distribution of this work must maintain attribution to the author(s) and the published article's title, journal citation, and DOI. Funded by SCOAP ${ }^{3}$. configuration for the $\left(3872,1^{+}\right)$, as described in [13] (see also [14]).

The discovery of two hidden charm pentaquarks gave further encouragement toward a deeper theoretical study of these particles. Here we assume that their spectrum may be described in terms of the chromomagnetic interaction (CMI), which has been successful in describing the mass differences between the octet $\frac{1}{2}^{+}$and the decuplet $3^{+}$[15]. The nonleptonic decays of the strange particles are described by the decay, see Fig. 1:

$$
s \rightarrow u+d+\bar{u}
$$

and the formation of the final hadrons by the products of the decay and by the spectators (a light antiquark for the kaons or two quarks for the strange baryons). In a similar way the production of hidden charm multiquarks is associated with the Cabibbo allowed process for the decaying $\Lambda_{b}$ with an amplitude proportional to $V_{c b}^{*} V_{c s}$ :

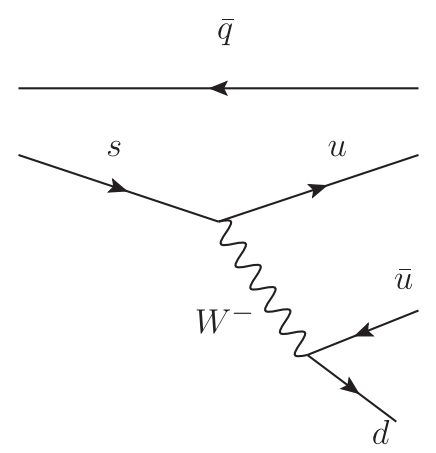

FIG. 1. Feynman diagram for the decay $\bar{K} \rightarrow \pi \pi$. 


$$
b \rightarrow c+s+\bar{c}
$$

followed by the emission of a gluon by the strange quarks, giving rise to a $q \bar{q}$ pair for the decay of $\bar{B}^{0}$ into $K_{S}^{0}+3872$, $1^{+}$, see Fig. 2., or to a $u \bar{u}$ pair for the decay into a $\mathrm{K}^{-}+$the hidden charm pentaquarks $4380,\left(\frac{3}{2}\right)^{-}$and 4450 , $\left(\frac{5}{2}\right)^{+}$discovered at $L H C_{b}$, see Fig. 3. The $1^{+}$resonance is built by the merging of the two $c \bar{c}$ and $q \bar{q}$ color octets of spin 1, while the pentaquarks by the union of the $c \bar{c}$ octet with the color and flavor octet with spin parity $\left(\frac{1}{2}\right)^{+}$built by the spectator diquark in the $\Lambda_{b}$ and the $\mathrm{u}$, produced by the gluon, while the $\bar{u}$ forms with the strange quark produced in the decay a $K^{-}$.

If the five constituents join in a relative $S$-wave, they may give rise to the $\frac{3-}{2}-$ hidden charm pentaquark, while if the two octets are in a $\mathrm{P}$-wave they may give rise to the $\frac{5}{2}^{+}$. In the first case, when they join, they give rise to a combination of eigenstates of the QCD Hamiltonian. In both cases isospin conservation requires that the three light quarks have $I=\frac{1}{2}$. Therefore, the Pauli principle demands that, if they are in S-wave with a symmetric wave function, they transform as the 70 representation of $S U(6)$ color spin, $S U(6)_{c s}$ (for three objects, a mixed symmetry state may give rise to an antisymmetric one only by multiplying it by

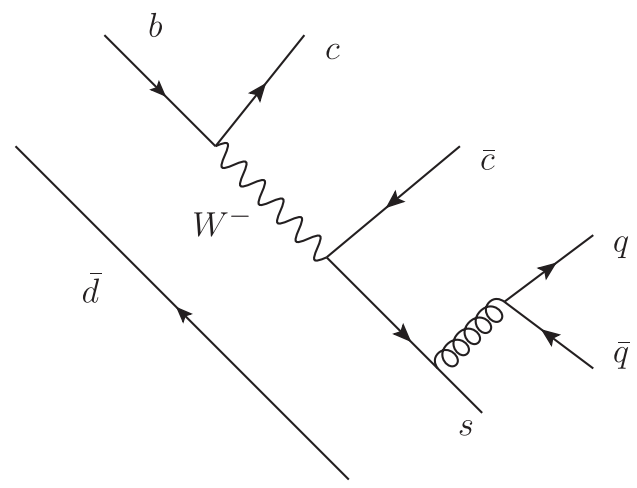

FIG. 2. Feynman diagram for the decay $\bar{B}^{0} \rightarrow \bar{K}^{0}+\left(3872,1^{+}\right)$.

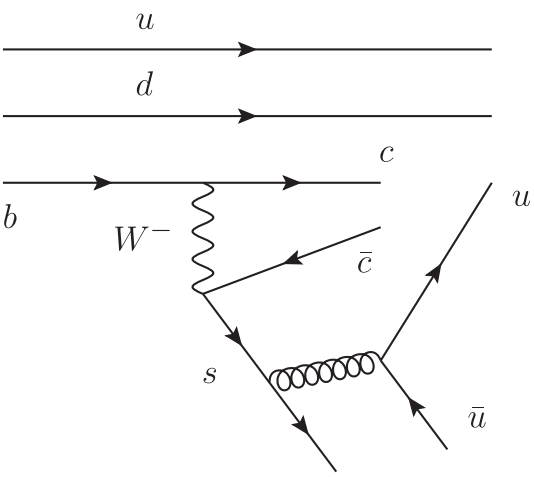

FIG. 3. Feynman diagram for the decay $\Lambda_{b} \rightarrow K^{-}+$Pentaquark with hidden charm. another mixed symmetry state). Otherwise, by multiplying it by a totally symmetric or antisymmetric state, one gets a mixed symmetry state [16-18]; while the $c \bar{c}$ pair transforms as the $35+1$ representation of $S U(6)_{c s}$.

The mechanism for the decay of the $\left(\frac{5}{2}\right)^{+}$pentaquark is similar to the decay of the $1^{+}$tetraquark at 3872 into $J / \psi+\rho^{0}$ (or $\left.\omega\right)$ : a gluon exchange, which turns the two color octets into singlets. Instead, their formation in the decay of $B_{q}$ and $\Lambda_{b}$ is different. In fact, in the first case the strange quark produced in the $b$ decay, forms a kaon together with the spectator antiquark, while the $q \bar{q}$ pair produced by the gluon forms together with the $c \bar{c}$ the $\left(3872,1^{+}\right)$tetraquark. The analogous process for the $\Lambda_{b}$ decay, with the strange quark forming with the spectator diquark a $\Lambda$, would give rise to the decay $\Lambda_{b} \rightarrow \Lambda+\left(3872,1^{+}\right)$, see Fig. 4.

Högaasen and Sorba [16] studied all the possibilities with three constituents in $\mathrm{P}$-wave with respect to the other two and came to the conclusion that the most interesting case is with two color octets of the three light quarks and the $c \bar{c}$ pair, with the caveat that each octet might be turned into an ordinary hadron by absorbing a gluon before combining to form the hidden charm pentaquark. One should keep in mind, however, the fact that, in the decay

$$
\Lambda_{b} \rightarrow p+J / \psi+K^{-},
$$

a gluon should be emitted and turned into a $(u \bar{u})$ pair to give rise to seven final constituents and therefore the presence of another gluon requires a higher order in QCD.

The seven constituents may also combine in a different way with the strange quark giving rise to a $\Lambda$ and the $c \bar{c}$ and the $u \bar{u}$ color octets to the $\left(3872,1^{+}\right)$. The decay

$$
\Lambda_{b} \rightarrow \Lambda+\left(3872,1^{+}\right)
$$

might be looked for in final states $p+\pi^{-}(\Lambda), \mu^{+} \mu^{-}(J / \psi)$, and $\pi^{+} \pi^{-}\left(\rho^{0}\right)$.

As we shall stress in the following sections, the "beautiful" particles, due to their relative long lifetime, decay at a distance from the interaction point sufficient enough to avoid the presence of the gluons emitted there.

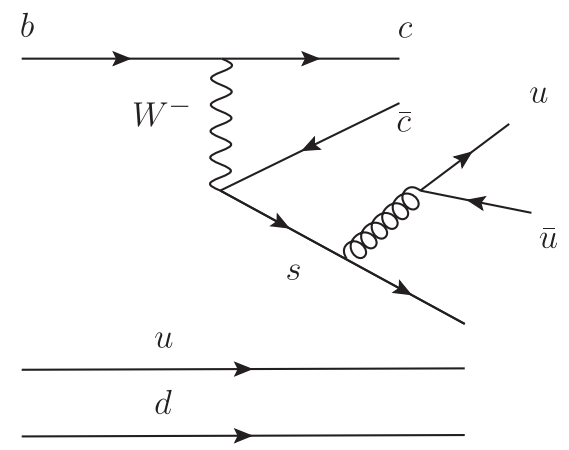

FIG. 4. Feynman diagram for the decay $\Lambda_{b} \rightarrow \Lambda+\left(3872,1^{+}\right)$. 
In the next section, we shall show the role of the chromomagnetic interaction (the fine structure term) to describe the spectrum of the ordinary hadrons and of the two lowest scalar nonets and the mass of the doubly charmed baryon recently discovered at $L H C b$ [19]. In the third section, we shall compute the masses of the two hidden charm pentaquarks. Our description will account for their different widths. In the fourth section, we shall give reasons why only some of the multiquark states have been detected. Then, we shall give our conclusions. In the Appendix, we describe some needed Clebsch-Gordon coefficients of $S U(6)$ and several identities that are useful in computing the chromo-magnetic contributions and the values of the quadratic Casimir operators of $S U(n)$, which appear in the evaluation of the chromomagnetic contribution to the mass pf the states.

\section{SPECTRUM OF THE LOWER (干) PARITY MESONS DESCRIBED BY CMI}

Accepting the proposal that QCD is the theory of strong interactions [20], De Rujula, Georgi, and Glashow [15] realized that the fine structure (the $\mathrm{CMI}$ ) accounts for the mass differences between $\Delta$ and the nucleon and between $\Sigma$ and the $\Lambda$. In this framework, there is the successful prediction

$$
M\left(\Xi^{*}\right)-M(\Xi)=M\left(Y^{*}\right)-M(\Sigma)
$$

which had been previously obtained, by assuming the same coefficients for the terms transforming both as an octet for the decuplet and the octet of baryons. Applying the same approach to the charmed baryons $\Sigma_{c}$ and $\Lambda_{c}$, they predicted a mass difference high enough to allow the strong decay $\Sigma_{c}^{+} \rightarrow \Lambda_{c}+\pi^{+}$, in agreement with the discovery of both particles in a neutrino experiment [21]. To find the spectrum of the lowest positive parity baryons, one considers the chromomagnetic interaction between the quarks $q_{1}$ and $q_{2}$, which is proportional to the product of the generators of $S U(6)_{c s}$ with a negative factor:

$$
\frac{\vec{\sigma} \lambda_{a}}{2}\left|q_{1}\right\rangle \times \frac{\vec{\sigma} \lambda_{a}}{2}\left|q_{2}\right\rangle
$$

The identity holds:

$$
\frac{\vec{\sigma} \lambda_{a}}{2} \times \frac{\vec{\sigma} \lambda_{a}}{2}=C_{6}-\frac{C_{3}}{2}-\frac{C_{2}}{3}
$$

where $C_{6}, C_{3}$, and $C_{2}$ are the quadratic Casimir of $S U(6)_{c} s$, $S U(3)_{c}$, and $S U(2)_{s}$, respectively [16,17]. Since the color singlets built with three quarks with spin $\frac{1}{2}$ and $\frac{3}{2}$ belong to the 70 and 20 representations of $S U(6)_{c s}$, respectively, the r.h.s. of Eq. (6), $C_{6}-\frac{C_{3}}{2}-\frac{C_{2}}{3}$, applied to the $70,1, \frac{1}{2}$ of $S U(6)_{c s}, S U(3)_{c}, S U(2)_{s}$ gives a factor 8 , to the $20,1, \frac{3}{2}$ a factor 4 and to the $6,3, \frac{1}{2}$ a factor 2 , we deduce that the factor needed to get the mass differences is $\frac{M_{N}-M_{\Delta}}{4}$. To get the masses of $N$ and $\Delta$, one has to add the contribution of the effective mass of the constituents, which is given by $\frac{M_{N}+M_{\Delta}}{2}$, since the chromomagnetic contributions to their masses are opposite.

To get the masses of the charmed baryons one has to keep into account that the chromomagnetic interaction between a light and a charmed quark is weaker, mainly for the smaller gyro-chromomagnetic factor of the charmed quark inversely proportional to its mass. With respect to the case of the light quarks to reproduce the masses a factor $k_{1}=0.24$ is needed for the CMI and an effective mass for the charmed quark of $1715 \mathrm{MeV}$.

The $\Sigma_{b}$ and $\Lambda_{b}$ particles have a mass difference even larger, as expected.

For the mesons $\left(\pi, K \rho, K^{*}\right)$, one should consider the CMI of a $q_{1}$ and a $\bar{q}_{3}$, which is proportional with a positive coefficient to:

$$
\frac{\vec{\sigma} \lambda_{a}}{2}\left|q_{1}\right\rangle \times \frac{\vec{\sigma} \lambda_{a}}{2}\left|\bar{q}_{3}\right\rangle
$$

The vector and the pseudoscalar mesons belong to the 35 and to the 1 representations of $S U(6)_{c s}$, respectively, and therefore the second member of Eq. (6), $C_{6}-\frac{C_{3}}{2}-\frac{C_{2}}{3}$, applied to them, gives the factors $\frac{16}{3}$ and 0 , respectively. Therefore the factor, which multiplies $C_{6}-\frac{C_{3}}{2}-\frac{C_{2}}{3}-4$ to get the chromomagnetic contribution for the light $q \bar{q}$ is $\frac{3\left(M_{\rho}-M_{\pi}\right)}{16}$, while the sum of their constituent masses is given by $\frac{3 M_{\rho}+M_{\pi}}{4}$. So one needs a larger chromomagnetic interaction and a smaller effective masses for the light and the strange quarks than in the case of the baryons. Both these properties can be understood by the more intense chromoelectric attraction between a quark and an antiquark, which form a color singlet with respect to two quarks, which combine in a $\overline{3}$ of $\mathrm{SU}(3)$ color. Indeed, the stronger attraction implies a smaller constituent mass and a larger contact interaction. In fact, for the charmed mesons $D$ and $D^{*}$, a slightly smaller mass, $1615 \mathrm{MeV}$, and larger $k_{c}=$ 0.26 are needed with respect to the charmed baryons. Also, the values found for the $c \bar{c}$ states, $1535 \mathrm{MeV}$ for the mass of the charmed quark and $K_{c}^{2}=0.186$ for the square of the gyro chromo-magnetic factor can be understood as a consequence of the smaller distance between the constituents. A mass of $3621.40 \mathrm{MeV}$ of the $\Xi_{c c}^{++}$recently found by $L H C b$ [19] implies an effective mass of the constituent charmed quarks of $1665 \mathrm{MeV}$, somewhat smaller than the one found for the charmed mesons and $\Lambda_{c}$.

For the two nonets of scalar tetraquarks, where the states built with the light constituents are the $f^{0}(600)$ and $f^{0}(1370)$, their masses are reproduced with an effective chromomagnetic interaction as for the baryons and with a 
larger constituent mass. Interestingly enough, this explains why the lowest one, which decays into two pions, has a very large width, while the other one decays mainly into four pions [22]. In fact, the $S U(6)_{c s}$ Casimir, which gives the most important chromomagnetic contribution to the masses, implies that the lighter state is almost a $S U(6)_{c s}$ singlet with an "open channel" [23] into two pions, which are also $S U(6)_{c s}$ singlets, while the heavier one transforms mainly as a 405 and therefore has an open channel into a pair of $\rho$ mesons, which transform as a 35 of $S U(6)_{c s}$ color spin $[14,24]$. We may be confident that also the pentaquark states are eigenvectors of the chromomagnetic interaction. The dependance of both the constituent masses and of the normalization of the CMI contributions to the mass splitting on the number of constituents in s-wave will be kept in account, when we shall study the spectrum of the pentaquark states. A general analysis of the spectrum of negative and positive pentaquarks built with the three lightest quarks can be found in [25] and the study of $3 q 3 \bar{q}$ hexaquarks in [26].

\section{FORMATION, MASSES, AND DECAYS OF THE HIDDEN CHARM PENTAQUARKS}

The Cabibbo allowed process for the decaying $\Lambda_{b}$ described in (2) is induced by the term of the nonleptonic lagrangian proportional to:

$$
\overline{c_{L}} \gamma_{\mu} b_{L} \overline{s_{L}} \gamma^{\mu} c_{L}
$$

which is a combination of

$$
\overline{c_{L}} \gamma_{\mu} c_{L} \overline{s_{L}} \gamma^{\mu} b_{L}
$$

and

$$
\overline{c_{L}} \gamma_{\mu} \lambda_{a} c_{L} \overline{s_{L}} \gamma^{\mu} \lambda_{a} b_{L}
$$

as a consequence of the $S U(3)$ crossing relations. While the term in Eq. (9) gives rise to the amplitude for the "golden channel" for $C P$ violation $(\bar{B})^{0} \rightarrow J / \psi+K_{S}^{0}$, the term in Eq. (10) is the first step for the decay $\Lambda_{b} \rightarrow J / \psi+p+K^{-}$. In fact the emission by the strange quark of a gluon converted into a $u \bar{u}$ pair completes the number of constituents, seven, needed to hadronize into the final particles. The scale, at which the gluon is emitted and converted needs not to be high and therefore the factor $\alpha_{s}$ needs not to be too small (an example, where a gluon is emitted and converted in a pair of light quarks is the decay $K \rightarrow \pi \pi \pi$ ). The produced $c$ quark, if it does not recombine with the spectator diquark $u d$ to give a $\Lambda_{c}$, may form a color octet with spin 1 with the $\bar{c}$. The $\bar{u}$ produced by the gluon may combine with the $s$-quark to form the negative kaon, while the $u$ with the spectator diquark in the $\Lambda_{b}$ may form a color octet with spin $\frac{1}{2}$ and the same flavor of the proton. The two color octets may give rise to one or the other of the two resonances, depending on their relative orbital momentum, the one with negative parity for the S-wave, the one with positive parity for the $\mathrm{P}$-wave. With all the constituents in $\mathrm{S}$-wave, one has four states with $S=\frac{3}{2}$, which can be obtained by the products $\frac{3}{2} \times 1, \frac{3}{2} \times 0$, and $\frac{1}{2} \times 1$. Let us remember that the 70 contains both $\frac{3}{2}$ and $\frac{1}{2}$ spin color octets and a spin $\frac{1}{2}$ singlet, while the 35 contains both 1 and 0 spin color octets and a spin 1 color singlet. So we have the following possibilities for the color-spin transformation properties of the three light quarks and the $c \bar{c}$ pair:

$$
\begin{aligned}
& \left(8, \frac{3}{2}\right) \times(8,1) \text { combined into a }\left(1, \frac{3}{2}\right) \\
& \left(8, \frac{3}{2}\right) \times(8,0) \text { combined into a }\left(1, \frac{3}{2}\right) \\
& \left(8, \frac{1}{2}\right) \times(8,1) \text { combined into a }\left(1, \frac{3}{2}\right) \\
& \left(1, \frac{1}{2}\right) \times(1,1) \text { combined into a }\left(1, \frac{3}{2}\right)
\end{aligned}
$$

The total contribution of CMI is given by:

$$
M=M(70)+M(70 \times 6)+M(70 \times \overline{6})+M(6 \times \overline{6}) .
$$

The four terms give the contribution of the chromomagnetic interaction of the three light quarks, of their interaction with the $c$ quark and the $\bar{c}$ antiquark and of the interaction of the two heavy constituents, $c$ and $\bar{c}$, respectively. The first and the fourth terms of Eq. (15) are given by:

$M(70)=-\frac{m_{\Delta}-m_{N}}{4}\left[C(70)_{6}-\frac{C(3 q)_{3}}{2}-\frac{C(3 q)_{2}}{3}-6\right]$

and

$$
\begin{aligned}
M(6 \times \overline{6})= & \frac{3}{16}\left(M_{J / \psi}-M \eta_{c}\right) \\
& \times\left[C(35)_{6}-\frac{C(c \bar{c})_{3}}{2}-\frac{C(c \bar{c})_{2}}{3}-4\right] .
\end{aligned}
$$

One has also to consider the chromomagnetic interaction between the charmed and the light quarks and their different chromomagnetic factors $[17,27]$. The sum of the contributions of the first and the fourth terms to the states defined in Eq. (11)-Eq. (14) is given by:

$$
M(70)+M(6 \times \overline{6})=\frac{m_{\Delta}-m_{N}}{8}-\frac{m_{J / \psi}-m_{\eta_{c}}}{32}
$$

for eigenstate (11), 
$M(70)+M(6 \times \overline{6})=\frac{m_{\Delta}-m_{N}}{8}+\frac{3\left(m_{J / \psi}-m_{\eta_{c}}\right)}{32}$

for eigenstate (12),

$M(70)+M(6 \times \overline{6})=-\frac{M_{\Delta}-M_{N}}{4}-\frac{M_{J / \psi}-M_{\eta_{c}}}{32}$

for eigenstate (13), while for the "open channel" (14) the sum is

$M(70)+M(6 \times \overline{6})=\frac{M_{N}-M_{\Delta}}{2}+\frac{M_{J / \psi}-M_{\eta_{c}}}{4}$.

The second and the third term in Eq. (13) are related to the chromomagnetic interaction of the light quarks with $c$ and $\bar{c}$, and are proportional to $k_{1}=0.24$ and $k_{2}=0.26$, the values of $k_{c}$ for charmed baryons and mesons, respectively. To evaluate them one should consider the tensor products:

$$
\begin{aligned}
& 70 \times 6=210+105+105^{\prime} \\
& 70 \times \overline{6}=384+21+15
\end{aligned}
$$

and the fact that

(i) the $(3,5)$ of $S U(6)_{c s}$ is contained in the $105^{\prime}$,

(ii) the three $(3,3)$ in the three representation of the first product,

(iii) one of the $(\overline{3}, 3)$ is contained in the 15 ,

(iv) the $(\overline{3}, 5)$ and the other two $(\overline{3}, 3)$ 's in the 384 for the second product.

Therefore the contributions of the second term is given by:

$$
\begin{aligned}
& \frac{k_{1}\left(M_{N}-M_{\Delta}\right)}{4}\left[C(3 q c)_{6}-\frac{C(3)_{3}}{2}-\frac{C(3 q c)_{2}}{3}\right. \\
& \left.-C(70)_{6}+\frac{C(3 q)_{3}}{2}+\frac{C(3 q)_{2}}{3}-2\right]
\end{aligned}
$$

while the contributions of the third term is given by:

$$
\begin{aligned}
& \frac{3 k_{2}\left(M_{\rho}-M_{\pi}\right)}{16}\left[C(3 q \bar{c})_{6}-\frac{C(\overline{3})_{3}}{2}-\frac{C(3 q \bar{c})_{2}}{3}\right. \\
& \left.-C(70)_{6}+\frac{C(3 q)_{3}}{2}+\frac{C(3 q)_{2}}{3}-2\right] .
\end{aligned}
$$

In conclusion, the terms proportional to $\left(M_{\Delta}-M_{N}\right)$ and to $\left(M_{\rho}-M_{\pi}\right)=\frac{1}{K_{c}^{2}}\left(M_{J / \psi}-M_{\eta_{c}}\right)$ are the matrices respectively shown in Tables I and II, while in Table III we show the numerical evaluation of Eq. (15) in $\mathrm{MeV}$ in the base of the states.
TABLE I. CMI contributions proportional to $\left(M_{\Delta}-M_{N}\right)$.

\begin{tabular}{lccc}
\hline \hline$\frac{1+3 k_{1}}{8}$ & 0 & 0 & 0 \\
0 & $\frac{1-3 k_{1}}{8}$ & $\frac{k_{1}}{3}$ & $\frac{k_{1}}{6}$ \\
0 & $\frac{k_{1}}{3}$ & $-\frac{1+3 k_{1}}{8}$ & $\frac{k_{1}}{6}$ \\
0 & $\frac{k_{1}}{6}$ & $\frac{k_{1}}{6}$ & $\frac{k_{1}-1}{2}$ \\
\hline \hline
\end{tabular}

TABLE II. CMI contributions proportional to $\left(M_{\rho}-M_{\pi}\right)$.

\begin{tabular}{lccc}
\hline \hline$\frac{3\left(-9 k_{2}+K_{c}^{2}\right)}{64}$ & $-\frac{\sqrt{15}\left(3 k_{2}+K_{c}^{2}\right)}{64}$ & $-\frac{\sqrt{15} k_{2}}{8}$ & $-\frac{\sqrt{15} k_{2}}{16}$ \\
$-\frac{\sqrt{15}\left(3 k_{2}+K_{c}^{2}\right)}{64}$ & $-\frac{\left(15 k_{2}+K_{c}^{2}\right)}{64}$ & $-\frac{\sqrt{15} k_{2}}{8}$ & $-\frac{\sqrt{15} k_{2}}{16}$ \\
$-\frac{\sqrt{15} k_{2}}{8}$ & $-\frac{k_{2}}{8}$ & $-\frac{\left(3 k_{2}-K_{c}^{2}\right)}{32}$ & $-\frac{k_{2}}{8}$ \\
$-\frac{\sqrt{15} k_{2}}{16}$ & $-\frac{k_{2}}{16}$ & $-\frac{k_{2}}{8}$ & $\frac{K_{c}^{2}}{4}$ \\
\hline \hline
\end{tabular}

TABLE III. Numerical evaluation of Eq. (12).

\begin{tabular}{lcrc}
\hline \hline 10.5 & -33 & -71.5 & -35.05 \\
-33 & 28.75 & 5.3 & 2.65 \\
-71.1 & 5.3 & -53.2 & 6.5 \\
-35.05 & 2.65 & 6.5 & -86.2 \\
\hline \hline
\end{tabular}

$$
\begin{aligned}
& |1\rangle=|70 \times 6,(8,4) \times(3,2) \rightarrow(3,5)\rangle \\
& |2\rangle=|70 \times 6,(8,4) \times(3,2) \rightarrow(3,3)\rangle \\
& |3\rangle=|70 \times 6,(8,2) \times(3,2) \rightarrow(3,3)\rangle \\
& |4\rangle=|70 \times 6,(1,2) \times(3,2) \rightarrow(3,3)\rangle
\end{aligned}
$$

with eigenvalues: $-120,-71,11$ and 80 in correspondence to the eigenvectors:

$(.057, .08, .59, .624)$

$(.225, .063, .604,-.762)$

$(.39, .847,-.35,-.094)$

$(.736,-.522, .-.041,-.15)$

The "open channel" $p+J / \psi$ has negligible components along the two eigenvectors corresponding to the two higher eigenvalues and substantial ones along the two lower ones. This agrees well with the mass of the $\left(\frac{3}{2}\right)^{-}$state if we take the constituent masses of the light quarks from the lowest baryons, and those of $c$ and $\bar{c}$ from the $\Lambda_{c}$ and from the lowest charmed mesons. Namely, extracting the constituents masses from:

$$
\frac{M_{\Delta}-M_{N}}{2}+M_{\Lambda_{c}}+\frac{3 M_{D^{*}}+M_{D}}{4}=4480 \mathrm{MeV}
$$

implies for the two lightest $\left(\frac{3}{2}\right)^{-}$states a mass of 4360 and $4409 \mathrm{MeV}$. It can be noticed that by taking the masses of charmed constituents from charmonium would lead to smaller constituent masses, but the presence of the three light quarks favors us to consider charmed baryon and mesons. 
The tendency of larger constituent masses with the increasing number of constituents in relative $\mathrm{S}$-wave may give rise to a larger global constituent mass. Indeed, the $Q^{2}$ dependence of the strong coupling constant, decreasing with the scale, might be an explanation for the different values of the constituent masses for the ordinary mesons and baryons, as well as for the different value at the scale of the negative parity states built with all the constituents $(3 q, c$, and $\bar{c})$ in $\mathrm{S}$-wave.

Also, for the lowest scalar tetraquarks built with light constituents, the effective masses of the constituents is about $400 \mathrm{MeV}$, larger than in the case of ordinary baryons.

The value found here has an important consequence, as it predicts two higher $\left(\frac{3}{2}\right)^{-}$states at 4491 and $4560 \mathrm{MeV}$. By considering $q q c-\bar{c} q$ combinations, the "open channels" $\left(\Lambda_{c} \bar{D}^{* 0}\right)$ and the $I=\frac{1}{2}$ combination $\frac{1}{\sqrt{3}}\left(\sqrt{2} \Sigma_{c}^{++} D^{\bar{*}-}-\right.$ $\left.\Sigma_{c}^{+} \bar{D}^{-0}\right)$ have different components along the CMI eigenvectors. While $\Lambda_{c} \bar{D}^{* 0}$ with total spin $\frac{1}{2}$ for the light quarks is a combination of the two last vectors and therefore has substantial components along the two lower mass eigenstates, $\frac{1}{\sqrt{3}}\left(\sqrt{2} \Sigma_{c}^{++} \bar{D}^{\bar{*}}\right)-\Sigma_{c}^{+} \overline{D^{* 0}}$ has components mainly along the two states with spin $S(u u d)=\frac{3}{2}$ for the light quarks, as can be seen from the identity for the states with $S=\frac{3}{2}$ :

$$
\begin{aligned}
\mid S(u u)= & \left.1, S(u u c)=\frac{1}{2}, S(c \bar{c})=1\right\rangle \\
= & \frac{1}{3}\left[\sqrt{5}\left|S(u u d)=\frac{3}{2},\right| S(c \bar{c})=1\right\rangle \\
& \left.-\sqrt{3} \mid S(\text { uud })=\frac{3}{2} S(c \bar{c})=0\right\rangle \\
& \left.+\left|S(u u d)=\frac{1}{2}, S(c \bar{c})=1\right\rangle\right]
\end{aligned}
$$

The relationship between the $(8 \times 8)_{1}$ and $1 \times 1$ for $(u u d)$ $c \bar{c}$ and $(u u c) d \bar{c}$ is supplied by the well-known $S U(3)$ identities:

$$
\begin{aligned}
\delta_{\alpha}^{\beta} \delta_{\gamma}^{\epsilon} & =\frac{1}{3} \delta_{\alpha}^{\epsilon} \delta_{\gamma}^{\beta}+\frac{1}{2}\left(\lambda_{a}\right)_{\alpha}^{\epsilon}\left(\lambda_{a}\right)_{\gamma}^{\beta} \\
\left(\lambda_{a}\right)_{\alpha}^{\beta}\left(\lambda_{a}\right)_{\gamma}^{\epsilon} & =\frac{16}{9} \delta_{\alpha}^{\epsilon} \delta_{\gamma}^{\beta}-\frac{1}{3}\left(\lambda_{a}\right)_{\alpha}^{\epsilon}\left(\lambda_{a}\right)_{\gamma}^{\beta}
\end{aligned}
$$

The fact that the chromomagnetic interaction for the light quarks (the ones with the higher gyro-chromomagnetic factor) gives a positive contribution to the state $\Sigma_{c} \bar{D}^{*}$ and negative for $\Lambda_{c} \bar{D}^{0 *}$ (in analogy with the large difference $\left.M_{\Sigma_{c}}-M_{\Lambda_{c}}[15,21]\right)$ leads us to guess that the $\Sigma_{c} \bar{D}^{*}$ and $\Lambda_{c}$ $\bar{D}^{0 *}$ open channels have large components along the 4560 and $4360 \mathrm{MeV}$ resonances, respectively. However, according to the formation mechanism starting from the third state $\left(8, \frac{1}{2}\right) \times(8,1)$, which has a negligible component along the higher eigenstate, the $\Sigma_{c} \bar{D}^{*}$ decay may be more easily seen for the 4491 resonance. For the decay of the $\Sigma_{c}^{++}$we may have the same sequence, namely:

$$
\Sigma_{c}^{++} \rightarrow \Lambda_{c}+\pi^{+}, \quad \Lambda_{c} \rightarrow p+K^{-}+\pi^{+}
$$

two reactions which have led to the discovery of $\Sigma_{c}^{++}$in a neutrino experiment [21].

Let us consider the chromomagnetic contributions for the two octets in P-wave, which build the $4450 \mathrm{MeV}, \frac{5}{2}+$ resonance For the three light quarks transforming as the 70 of $S U(6)_{c s}$ the chromomagnetic contribution depends on their color and their spin:

$M(70)=-\frac{m_{\Delta}-m_{N}}{4}\left[C(70)_{6}-\frac{C(3 q)_{3}}{2}-\frac{C(3 q)_{2}}{3}-6\right]$

For the $c \bar{c}$ pair one has:

$$
\begin{aligned}
& M(6 \times \overline{6}) \\
& =\frac{3}{16}\left(M_{J / \psi}-M \eta_{c}\right)\left[C(35)_{6}-\frac{C(c \bar{c})_{3}}{2}-\frac{C(c \bar{c})_{2}}{3}-4\right]
\end{aligned}
$$

The sum of the two contributions is

$$
-\frac{m_{\Delta}-m_{N}}{8}-\frac{M_{J / \psi}-M \eta_{c}}{32}
$$

which sum up to $-40 \mathrm{MeV}$. We expect larger constituent masses for the two octets, since the chromoelectric interaction is less actractive for the three light quarks and slightly repulsive for the $c \bar{c}$ pair. Of course this is in part compensated by the attraction of the two color octets in P-wave. Also we expect a positive contribution from the rotational energy and the spin-orbit term for the three light quarks (An analogy may be advocated with the $1675, \frac{5}{2}-$ isospin $\frac{1}{2}$ resonance, classified in the $70, L=1$ of $S U(6)_{f s} \times S O(3)$, thought as a diquark of spin 1 and a quark in P-wave, with a mass larger than $\frac{M_{\Delta}+M_{N}}{2}$ by a large amount).

The narrow width of the $4450, \frac{5}{2}+$ can be explained by the fact that the decay into $p+J / \psi$ needs the exchange of one gluon, as it happens for the decay of the $\left.(3872), 1^{+}\right)$into $J / \psi+\rho^{0}$ (or $\omega$ ), if one identifies it as the state built with the light $(q \bar{q})$ and the charmed $(c \bar{c})$ pairs transforming as the $(8,3)$ representation of $S U(3)_{c} \times S U(2)_{s}$ [13].

The mechanism of formation of the strange isoscalar pentaquark with hidden charm is similar to the description of the formation of the $38721^{+}$in B decays, with the strange quark produced in the weak decay forming a strange color octet together with the scalar and isoscalar spectator in $\Lambda_{b}$. 
Notice that, in general, it is not easy to produce hadrons with nonminimal number of constituents, since the $q$ and $\bar{q}$ produced by the gluons tend to combine very fast into color singlets and the easiest way is to form ordinary hadrons. In Cabibbo allowed B decays, the creation of a $c \bar{c}$ color octet pair, which exerts an attraction on another octet built with a $q \bar{q}$ pair or three light quarks, can give rise to hadron states with hidden charm.

In conclusion, the interpretation of the two pentaquark resonances with hidden charm, discovered at $L H C b$ [1], as built with a $c \bar{c}$ and three light quark color octets in S-wave for the $\left(\frac{3}{2}\right)^{-}$, and with the five constituents in P-wave for the $\left(\frac{5}{2}\right)^{+}$, accounts for their different widths. An important consequence of this description is the prediction of two $\left(\frac{3}{2}\right)^{-}$ resonances at a mass of 4360 and $4560 \mathrm{MeV}$, with large components along the open channels $\Lambda_{c} \bar{D}^{+0}$ and $\Sigma_{c} \bar{D}^{*}$ final states, respectively.

As we shall stress in the following section, due to their relative long lifetimes the "beautiful" particles decay at a distance from the interaction point sufficient enough to avoid the presence of the gluons emitted there. Such gluons give rise to $q \bar{q}$ pairs transforming as color octets with the $q$ 's and the $\bar{q}$ 's, which build ordinary hadrons with the other constituents.

\section{FORMATION OF MULTIQUARK STATES}

The fact that the $\left(3872,1^{+}\right)$, which is a compact object since it is produced also at high $p_{T}$, unlike the deuteron, and is seen only through its neutral component, shows the relevance of the formation of multiquark states. In fact, the mechanism described in the previous sections is operative only for the neutral component, which is indeed the only component discovered.

As for the states predicted by Jaffe [23], strong evidence concerns the two scalar nonets, namely:

(i) the multiplet consisting of $f^{0}(600), \kappa(770)$ and $f^{\prime 0}$ and $a^{0}$, degenerate as expected, at $980 \mathrm{MeV}$

(ii) another one for which $f^{0}(1370)$ is the one consisting of light constituents.

As discussed before, our interpretation is that this second scalar nonet transforms almost like a 405 of $S U(6)_{c s}$, and thus it has an open channel into two $\rho$ 's, which transform as a $35(35 \times 35=1+\ldots 405)$. Albeit being below threshold, the large $\rho$ width makes the $f^{0}(1370)$ appear in 4 pions, as follows from the analysis in [22].

The fact that tetraquarks with light constituents and higher spin have not yet been seen led the Rome group [28] to consider only the diquarks transforming as $(\overline{3}, S=0, \overline{3})$ with respect to $S U(3)_{c} \times S U(2)_{s} \times S U(3)_{f}$ and their antiparticles, which may give rise to only one scalar nonet. To account for the heavier one, they advocate an instanton [29]. As we have shown in the second section, the masses and decays of the two states built with the light constituents are well described by deducing their spectrum with the same approach followed in [15] for ordinary hadrons. In fact, when the $\overline{3}, S=0$ and $3, S=0$ join, they give rise to a superposition of eigenstates of the CMI, with open channel [23] two pions or two $\rho$ 's, respectively.

To build the $\left(3872,1^{+}\right)$, the Rome group considered also diquarks transforming as a $(\overline{3}, 3)$ under $S U(3)_{c} x S U(2)_{s}$ [30]. For these diquarks the chromoelectric force is attractive, while the chromomagnetic is repulsive, which makes their formation less probable. Moreover, as well as the diquark $(\overline{3}, 1)$, they may combine with a quark to form a baryon. The $(\overline{3}, 3)$ may also give rise to a flavor decuplet and therefore a lower limit to the ratio of the abundances of $(\overline{3}, 3)$ and $(\overline{3}, 1)$ may be given by the ratio of the nondiffractive production of $\Delta$ and $\mathrm{N}$.

Diquarks are considered to build tetraquarks [31] as well as pentaquarks [32] with a description different from the one presented here. The mechanism proposed here for the formation of the $\left(3872,1^{+}\right)$, which accounts for the fact that only its neutral component has been found, is according to us better motivated. The tendency of the diquark $(\overline{3}, 3)$ to form a baryon with the quark, rather than combine with a $(\overline{3}, 1)$ diquark to build a spin 1 state or with a $(\overline{3}, 3)$ diquark to give rise to spin and (or) isospin 2 states explains why the large class of states predicted by Jaffe has not yet been found. According to us, the approach introduced in [15] for ordinary baryons can be successfully extended to find the spectrum of multiquark states of ordinary mesons. However, a production mechanism is needed to prevent that their formation from being overwhelmed by the recombination of the $\mathrm{q}$ and $\bar{q}$ produced by the gluons into ordinary hadrons. For this reason, the decays of particles with beauty, produced at Belle and $B A B A R$, and also at $L H C b$, offer a favorable opportunity, since the beautiful particles decay in absence of associated production. This is evident for the $e^{+} e^{-}$ rings, but it happens also for the particles produced at $L H C b$, since the long lifetime of the $b$ quark allows the hadrons with beauty to leave the interaction point before decaying, with the consequence that the products of their decays are not surrounded by the $q \bar{q}$ pairs produced in the interaction.

As for the formation of the $\Xi_{c c}^{++}$previously mentioned [19], our interpretation is that it is probably due to the union of a $c u$ scalar diquark with a $c$ : while its decay into $\Lambda_{c}+$ $K^{-}+\pi^{+}+\pi^{+}$requires that the allowed Cabibbo decay is accompanied by the creation of both a $u \bar{u}$ and a $d \bar{d}$ pairs, the $\Lambda_{c} \rightarrow P+K^{-}+\pi^{+}$implies the formation of a $u \bar{u}$ pair.

\section{CONCLUSIONS}

The analysis presented in this paper leads us to the following conclusions:

(i) The approach based on the chromomagnetic interaction to find the spectrum of the multiquark states, 
applied successfully for the $\left(3872,1^{+}\right)$[13] and to the lowest scalar nonets [14], is bolstered by the discovery of the two hidden charm pentaquarks at $L H C b$; it also accounts for the different widths of the $\frac{3}{2}^{-}$and $\frac{5}{2}^{+}$resonances.

(ii) A confirmation of the description proposed here should be the detection of $\Lambda_{c} \bar{D}^{* 0}$ and $\Sigma_{c} \bar{D}^{*}$ final states.

(iii) The property of the beautiful particles of traveling away from the interaction point, as a consequence of their lifetime, prevents the formation of hidden charm multiquarks through the Cabibbo favored decay with the production of a pair $c \bar{c}$ from being overwhelmed by the production of ordinary hadrons.

\section{ACKNOWLEDGMENTS}

I am very grateful to Professor Mario Abud for his explanation of the nondetection of the charged partners of the 3872, which inspired the considerations on the relevance of the formation mechanism of multiquark states. I thank Professor Antonello Polosa for bringing Refs. [1012] to my attention. I am grateful to Professor Alvaro De Rujula for bringing Ref. [21] to my attention.

\section{APPENDIX: MATHEMATICAL PROPERTIES OF $S U(6), S U(3)$ AND $S U(2)$}

The evaluation of the contributions proportional to $k_{1}$, $k_{2}$, and $K_{c}^{2}$ require the knowledge of the following CG of $S U(6)$ color spin:

$$
\begin{aligned}
\left|105^{\prime}, 3, S=2\right\rangle=\mid & \left.70 \times 6,8 \times 3, \frac{3}{2} \times \frac{1}{2}\right\rangle \\
\left|105^{\prime}, 3, S=1\right\rangle= & \frac{1}{\sqrt{6}}\left[\left|70 \times 6,8 \times 3, \frac{3}{2} \times \frac{1}{2}\right\rangle\right. \\
& +\left|70 \times 6,8 \times 3, \frac{1}{2} \times \frac{1}{2}\right\rangle \\
& \left.+2\left|70 \times 6,1 \times 3, \frac{1}{2} \times \frac{1}{2}\right\rangle\right] \\
|105,3, S=1\rangle= & \frac{1}{\sqrt{3}}\left[\left|70 \times 6,8 \times 3, \frac{3}{2} \times \frac{1}{2}\right\rangle\right. \\
& +\left|70 \times 6,8 \times 3, \frac{1}{2} \times \frac{1}{2}\right\rangle \\
& \left.-\left|70 \times 6,1 \times 3, \frac{1}{2} \times \frac{1}{2}\right\rangle\right] \\
|210,3, S=1\rangle= & \frac{1}{\sqrt{2}}\left[\left|70 \times 6,8 \times 3, \frac{3}{2} \times \frac{1}{2}\right\rangle\right. \\
& \left.-\left|70 \times 6,8 \times 3, \frac{1}{2} \times \frac{1}{2}\right\rangle\right]
\end{aligned}
$$

$$
\begin{aligned}
&|384, \overline{3}, \bar{S}=2\rangle=\left|70 \times \overline{6}, 8 \times \overline{3}, \frac{3}{2} \times \frac{1}{2}\right\rangle \\
&\left|384_{1}, \overline{3}, \bar{S}=1\right\rangle=\frac{1}{\sqrt{105}}\left[5\left|70 \times \overline{6}, 8 \times \overline{3}, \frac{3}{2} \times \frac{1}{2}\right\rangle\right. \\
&-8\left|70 \times \overline{6}, 8 \times \overline{3}, \frac{1}{2} \times \frac{1}{2}\right\rangle \\
&\left.-4\left|70 \times \overline{6}, 1 \times \overline{3}, \frac{1}{2} \times \frac{1}{2}\right\rangle\right] \\
&\left|384_{2}, \overline{3}, \bar{S}=1\right\rangle= \frac{1}{\sqrt{5}}\left[\left|70 \times \overline{6}, 8 \times \overline{3}, \frac{1}{2} \times \frac{1}{2}\right\rangle\right. \\
&\left.-2\left|70 \times \overline{6}, \times \overline{3}, \frac{1}{2} \times \frac{1}{2}\right\rangle\right] \\
&|15, \overline{3}, \bar{S}=1\rangle= \frac{1}{\sqrt{21}}\left[4\left|70 \times \overline{6}, 8 \times \overline{3}, \frac{3}{2} \times \frac{1}{2}\right\rangle\right. \\
&+2\left|70 \times \overline{6}, 8 \times \overline{3}, \frac{1}{2} \times \frac{1}{2}\right\rangle \\
&\left.+1\left|70 \times \overline{6}, 1 \times \overline{3}, \frac{1}{2} \times \frac{1}{2}\right\rangle\right]
\end{aligned}
$$

where $S$ is the total spin of the three light quarks and of $c$ and $\bar{S}$ is the total spin of the three light quarks and of $\bar{c}$. If the spin of the light quarks and the total spin are both $\frac{3}{2}$ the following identities follow:

$$
\begin{aligned}
|S=2\rangle & =-\frac{1}{4}[|\bar{S}=2\rangle+\sqrt{15}|\bar{S}=1\rangle] \\
& =\frac{1}{\sqrt{8}}\left[\sqrt{3}\left|S_{c \bar{c}}=1\right\rangle+\sqrt{5}\left|S_{c \bar{c}}=0\right\rangle\right] \\
|S=1\rangle & =\frac{1}{4}[|\sqrt{15} \bar{S}=2\rangle-|\bar{S}=1\rangle] \\
& =\frac{1}{\sqrt{8}}\left[\sqrt{5}\left|S_{c \bar{c}}=1\right\rangle-\sqrt{3}\left|S_{c \bar{c}}=0\right\rangle\right]
\end{aligned}
$$

The Casimir of $S U(n)$ relevant for these work are

$$
\begin{aligned}
& C(6)_{6}=\frac{35}{12} \\
& C(15)_{6}=\frac{14}{3} \\
& C(20)_{6}=\frac{21}{4} \\
& C(21)_{6}=\frac{14}{3} \\
& C(35)_{6}=6
\end{aligned}
$$




$$
\begin{gathered}
C(56)_{6}=\frac{45}{4} \\
C(70)_{6}=\frac{33}{4} \\
C\left(105^{\prime}\right)_{6}=\frac{26}{3} \\
C(105)_{6}=\frac{32}{3} \\
C(210)_{6}=\frac{38}{3}
\end{gathered}
$$

$$
\begin{aligned}
C(384)_{6} & =\frac{35}{3} \\
C(3)_{3} & =\frac{4}{3} \\
C(8)_{3} & =3 \\
C(S)_{2} & =S(S+1)
\end{aligned}
$$

[1] R. Aaij et al. (LHCb Collaboration), Phys. Rev. Lett. 115, 072001 (2015).

[2] S. K. Choi et al. (Belle Collaboration), Phys. Rev. Lett. 91, 252001 (2003); D. Acosta et al. (CDF Collaboration), Phys. Rev. Lett. 93, 072001 (2004); V. M. Abazov et al. (D0 Collaboration), Phys. Rev. Lett. 93, 162002 (2004); B. Aubert et al. (BABAR Collaboration), Phys. Rev. D 71, 071103 (2005); R. A. Aaij et al. (LHCb Collaboration), Phys. Rev. Lett. 110, 222001 (2013).

[3] J. Rosner, Phys. Rev. Lett. 21, 950 (1968).

[4] R. L. Jaffe, Phys. Rev. D 15, 267 (1977).

[5] H. M. Chan and H. Högaasen, Phys. Lett. 76B, 634 (1978).

[6] R. L. Jaffe, Phys. Rev. Lett. 38, 195 (1977).

[7] V. A. Matveev and P. Sorba, Nuovo Cim. Soc. Ital. Fis. 20, 121 (1977); Nuovo Cim. Soc. Ital. Fis. 45A, 257 (1978).

[8] H. Högaasen and P. Sorba, Fiz. B 14, 245 (2005).

[9] Ya. I. Azimov, J. Phys. G 37, 023001 (2010); Proceedings of the International Workshop Hadron Structure and QCD, edited by V. T. Kim and L. V. Lipatov (Peterburgskiy Institut Yadernoy Fiziki Im. B. P. Konstantinov, Gatchina, St. Petersburg, 2012), 158.

[10] S. Chatchyan et al. (CMS Collaboration), J. High Energy Phys. 04 (2013) 154.

[11] A. Esposito, A. L. Guerrieri, L. Maiani, F. Piccinini, A. Pilloni, A. D. Polosa, and V. Riquer, Phys. Rev. D 92, 034028 (2015).

[12] J. Adam et al. (ALICE Collaboration), arXiv:1506.08453; arXiv:1506.08951; B. Abelev et al. (ALICE Collaboration), Phys. Rev. C 88, 044909 (2013).

[13] H. Högaasen, J. M. Richard, and P. Sorba, Phys. Rev. D 73, 054013 (2006).

[14] F. Buccella, H. Högaasen, J. M. Richard, and P. Sorba, Eur. Phys. J. C 49, 743 (2007).
[15] A. De Rújula, H. Georgi, and S. L. Glashow, Phys. Rev. D 12, 147 (1975).

[16] H. Högasen and P. Sorba, Nucl. Phys. B145, 119 (1978).

[17] M. de Combrugghes, H. Högaasen, and P. Sorba, Nucl. Phys. B156, 347 (1979).

[18] F. Buccella and P. Sorba, Mod. Phys. Lett. A 19, 1547 (2004).

[19] R. Aaij et al. (LHCb Collaboration), Phys. Rev. Lett. 119, 112001 (2017).

[20] H. Fritzsch, M. Gell-Mann, and H. Leutwyler, Phys. Lett. 47B, 365 (1973).

[21] E. G. Cazzoli, A. M. Cnops, P. L. Connolly, R. I. Louttit, M. S. Murtagh, P. A Palmer, N. P. Samios, T. T. Tao, and H. H. Williams, Phys. Rev. Lett. 34, 1125 (1975).

[22] M. Gaspero, Nucl. Phys. A562, 407 (1975).

[23] R. L. Jaffe, Phys. Rev. D 17, 1444 (1978).

[24] F. Buccella, Mod. Phys. Lett. A 21, 831 (2006).

[25] M. Abud, F. Buccella, D. Falcone, G. Ricciardi, and F. Tramontano, Adv. Stud. Theor. Phys. 2, 929 (2008).

[26] M. Abud, F. Buccella, and F. Tramontano, Phys. Rev. D 81, 074018 (2010).

[27] H. Högasen and P. Sorba, Nucl. Phys. B145, 119 (1978).

[28] L. Maiani, F. Piccinini, A. D. Polosa, and V. Riquer, Phys. Rev. Lett. 93, 212002 (2004).

[29] G. 't Hooft, G. Isidori, L. Maiani, A. D. Polosa, and V. Riquer, Phys. Lett. B 662, 424 (2008).

[30] L. Maiani, F. Piccinini, A. D. Polosa, and V. Riquer, Phys. Rev. D 71, 014028 (2005).

[31] L. Maiani, F. Piccinini, A. D. Polosa, and V. Riquer, Phys. Rev. D 89, 114010 (2014).

[32] L. Maiani, A. D. Polosa, and V. Riquer, Phys. Lett. B 749, 289 (2015). 